\title{
Survey of anti-Toxoplasma gondii antibodies in wild mammals captured from Atlantic Forest fragments in Bahia, northeastern Brazil
}

\author{
Pesquisa de anticorpos anti-Toxoplasma gondii em mamíferos silvestres em \\ áreas de Mata Atlântica da Bahia, nordeste do Brasil
}

Pedro de Alcântara Brito Junior; Josiane Moreira Rocha; Caroline Araújo da Silva²; Priscylla Marcelly Vilanova Oliveira²; Joelande Esquivel Correia; Luciara Alves da Cruz; Anaiá da Paixão Sevá1,3 (D); Téo Veiga de Oliveira² (1); Aristeu Vieira da Silva² (D); Martín Roberto del Valle Alvarez ${ }^{4}$ (D); George Rêgo Albuquerque ${ }^{5 *}$ (1)

\author{
'Programa de Pós-graduação em Ciência Animal - PPGCA, Universidade Estadual de Santa Cruz - UESC, Ilhéus, BA, Brasil \\ ${ }^{2}$ Departamento de Ciências Biológicas, Universidade Estadual de Feira de Santana - UEFS, Feira de Santana, BA, Brasil \\ ${ }^{3}$ Laboratório de Epidemiologia e Bioestatística, Faculdade de Medicina Veterinária e Zootecnia - FMVZ, Universidade de São Paulo - USP, \\ São Paulo, SP, Brasil \\ ${ }^{4}$ Departamento de Ciências Biológicas, Universidade Estadual de Santa Cruz - UESC, Ilhéus, BA, Brasil \\ ${ }^{5}$ Departamento de Ciências Agrárias e Ambientais, Universidade Estadual de Santa Cruz - UESC, Ilhéus, BA, Brasil
}

\begin{abstract}
How to cite: Brito Junior PA, Rocha JM, Silva CA, Oliveira PMV, Correia JE, Cruz LA, et al. Survey of anti-Toxoplasma gondii antibodies in wild mammals captured from Atlantic Forest fragments in Bahia, northeastern Brazil. Braz J Vet Parasitol 2020; 29(4): e004720. https://doi.org/10.1590/S1984-29612020083
\end{abstract}

\begin{abstract}
The objective of this study was to investigate the frequency of antibodies to Toxoplasma gondii present in wild mammals that were trap captured in forest fragments in the State of Bahia, northeastern Brazil. A total of 368 individuals (246 rodents, 104 marsupials and 18 bats) were captured using live catch traps. Serum samples were tested using the modified agglutination test, with a cut-off point at 1:25 dilution. The total occurrence of antibodies to $T$. gondii was $10.6 \%$ (39/368), being $16.3 \%$ (17/104) in marsupials, 8.5\% (21/246) in rodents, and $5.5 \%(1 / 18)$ in bats. Antibody titers varied between 25 and 50 for rodents, between 25 and 400 for marsupials, and were 25 for bats. This is the first report on antibodies to T. gondii in certain rodent species (Thaptomys nigrita, Hylaeamys laticeps, and Cerradomys subflavus), marsupial species (Monodelphis americana, Gracilinanus microtarsus, Gracilinanus agilis and Marmosops incanus), and bats of the genus Rhynchonycteris. The presence of antibodies to T. gondii in wild mammals demonstrates the possibility of these animals as sentinels of toxoplasmosis, especially on regions under high anthropogenic effect.
\end{abstract}

Keywords: Toxoplasmosis, wild animals, serology, MAT.

\begin{abstract}
Resumo
O objetivo deste trabalho foi investigar a frequência de anticorpos anti-Toxoplasma gondii presentes em mamíferos selvagens, capturados em fragmentos florestais do Estado da Bahia, Nordeste do Brasil. Um total de 368 indivíduos (246 roedores, 104 marsupiais e 18 morcegos) foram capturados, usando-se armadilhas de captura viva. Os soros foram testados pelo teste de aglutinação modificada, com ponto de corte na diluição de 1:25. A ocorrência total de anticorpos anti-T. gondii foi de 10,6\% (39/368), sendo 16,3\% (17/104) em marsupiais, 8,5\% (21/246) em roedores e $5,5 \%(1 / 18)$ em morcegos. Os títulos variaram de 25 a 50 e 25 a 400, respectivamente, para roedores e marsupiais, e o título máximo em morcegos foi de 25. Este é o primeiro relato de anticorpos para T. gondii em algumas espécies de roedores (Thaptomys nigrita, Hylaeamys laticeps e Cerradomys subflavus), em marsupiais (Monodelphis americana, Gracilinanus microtarsus, Gracilinanus agilis e Marmosops incanus) e em quiróptero do gênero Rhynchonycteris. A presença de anticorpos anti-T. gondii em mamíferos selvagens demonstra a possibilidade desses animais como sentinelas da toxoplasmose, principalmente em regiões com alto efeito antropogênico.
\end{abstract}

Palavras-chave: Toxoplasmose, animais silvestres, sorologia, MAT. 


\section{Introduction}

Atlantic Forest is one of the richest areas in terms of biodiversity and one of the most threatened areas on the planet, being considered a global conservation hotspot (Myers et al., 2000). Reduced to less than $8 \%$ of its original extension, Atlantic Forest stretches along the Brazilian coast, distributed across 17 States. Devastation of this biome is a result of human occupation and disordered exploitation of urban natural resources (Pinto et al., 2006). These environmental changes may trigger the appearance of zoonoses and emerging and reemerging diseases, which may lead to changes in wild animal populations, and alter the rates of parasitic infections and infestations (Sangioni et al., 2005; Thompson, 2013).

Wild living animals have been described as a source of parasitic diseases to humans and domestic animals (Thompson et al., 2010). One of these diseases is toxoplasmosis, a cosmopolitan parasitic zoonosis that can infect a wide variety of endothermic animals, including humans. Toxoplasmosis is caused by the protozoan Toxoplasma gondii, of the Sarcocystidae family of coccidia. The disease has an optional heteroxenic life cycle, with domestic and wild felids as the definitive hosts (Tenter et al., 2000).

Parasite dynamics are well understood in domestic animals and humans, but there is considerably less knowledge on the cycle in the wild. Thus, serological surveys should be carried out in order to scale the problem of toxoplasmosis, as the infected animals may indicate environmental contamination with oocysts (RendónFranco et al., 2014). It is important to rectify the shortcomings in this knowledge since the interaction between wildlife and urban populations is on the increase and threatens both species conservation and public health (Thompson et al., 2010; Rendón-Franco et al., 2014).

Small mammals such as rodents and marsupials play an important role in the life cycle of $T$. gondii, being a possible source of infection to domestic and wild cats, besides promoting the spread of the parasite in the environment (Gennari et al., 2015). Studies on the eating habits of wild cats suggest that rodents comprise about two-thirds of the consumed prey, although this can vary according to season, rodent availability, species of felid, and the availability of other prey (Rendón-Franco et al., 2014).

Toxoplasma gondii naturally infects several species of marsupials and rodents, however, the frequencies of such infections can vary according to the region and the diagnostic technique used. Studies in Brazil have shown an infection rate ranging from $5.7 \%$ to $61.1 \%$ in wild rodents and from $4.7 \%$ to $63.6 \%$ in marsupials (Ferraroni \& Marzochi, 1980; Salata et al., 1985; Yai et al., 2003; Cola et al., 2010, Costa et al., 2012; Vitaliano et al., 2014; Fournier et al., 2014; Gennari et al., 2015; Abreu et al., 2016; Horta et al. 2016). Research on this parasite in Chiroptera is even more scarce, although there are records in São Paulo (Cabral et al., 2013; 2014) showing more than $36.6 \%$ positives. In the State of Bahia, Jesus et al. (2017) found the presence of T. gondii DNA in 97 bat species, $2.1 \%$ representing positive samples.

In Brazil, and especially in the State of Bahia, the study of parasitic zoonoses in wild animals is still scarce. Therefore, this paper aims to determine the occurrence of antibodies to T. gondii in rodents, marsupials, and bats captured in fragments of Atlantic Forest in the State of Bahia, Northeast Brazil.

\section{Materials and Methods}

\section{Study area}

This study covered 27 areas of Atlantic Forest distributed across ten municipalities in the State of Bahia. These areas were grouped by geographic proximity, and named as Serra da Jiboia, Pratigi Environmental Protection Area (APA in the Portuguese abbreviation), South and Far South. According to vegetation, areas were classified into two groups, Atlantic Forest and Cabruca agroforestry systems. Cabruca is a local term used to designate the areas where cocoa is grown in areas shaded by native forest (Piasentin \& Saito, 2014), in this work the collection areas in the municipality of Ilheús. All the areas were georeferenced with the Global Positioning System (GPS) (Figure 1).

In the Serra da Jiboia (municipalities of Elísio Medrado, and Varzedo) and in the Pratigi APA (municipalities of Nilo Peçanha, Ituberá, Igrapiúna, and Ibirapitanga) the collections were conducted in three and nine areas, respectively. This region is a mountain chain with an almost north to south orientation, located in the eastern portion of the state of Bahia, Brazil, with around 5,500 ha still covered by two of the most typical Atlantic Forest environments, Dense Ombrophilous Forest and Semideciduous Seasonal Forest. The climate in the area is Tropical Semi-arid to Semi-humid, with a mean temperature of $23^{\circ} \mathrm{C}$ and mean annual rainfall of 1,200mm (Bahia, 2015). 

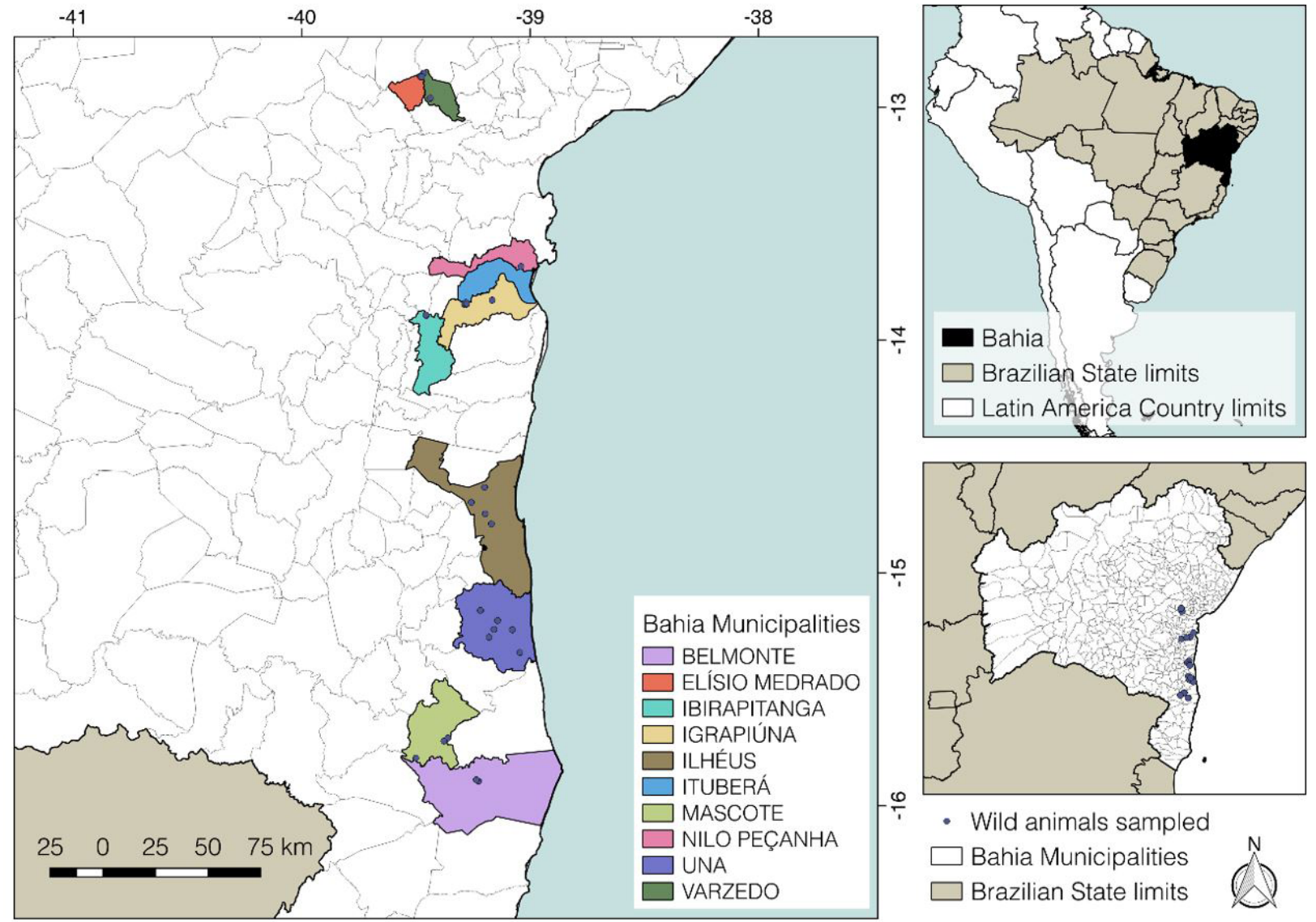

Figure 1. Wild mammal capture sites for Toxoplasma gondii antibody research study in municipalities in the state of Bahia, Brazil.

In Southern Bahia, municipality of Ilhéus, samples were collected in four locations of cocoa (Theobroma cacao) agroforestry systems (locally known as 'Cabruca'), and six located in forest fragments of the Atlantic Forest in the municipality of Una. Cabruca is a traditional agroforestry system in the region, which manages crops in the shade of the native trees of the Atlantic Forest. Are characterized by a landscape with few remnants of native vegetation and open areas (pastures, annual plantations and urban areas) (Faria et al. 2006).

In the far south, collections were made in five areas with forest remnants distributed by the municipalities of Mascote and Belmonte. The original landscape in this region is Lowland Rain Forest (Thomas, 2003), immersed in shaded cocoa, rubber tree, coffee, pastures, and eucalyptus. The climate of the region is hot and humid, with mean annual temperature of $25^{\circ} \mathrm{C}$ and mean annual rainfall between $1,200 \mathrm{~mm}$ and 1,800 $\mathrm{mm}$, evenly distributed throughout the year (Mori et al., 1983).

\section{Capture of animals}

To capture the small non-volant mammals, Sherman $(23 \times 8 \times 9 \mathrm{~cm}$ and $43 \mathrm{~cm} \times 14.5 \mathrm{~cm} \times 12.5 \mathrm{~cm})$ and Tomahawk $(32 \times 15 \times 15 \mathrm{~cm}, 50 \times 17 \times 17 \mathrm{~cm}$, and $51 \times 21 \times 21 \mathrm{~cm})$ live traps were used, in the same proportion both on the ground and in the understory, with 36 traps of each type per area. Furthermore, pitfall trap lines were added, consisting of $60 \mathrm{~L}$ plastic buckets, buried with the rim at ground level. Each line was composed of seven buckets placed $10 \mathrm{~m}$ apart and connected by a guiding fence about $50 \mathrm{~cm}$ in height, totaling 21 pitfall traps per area.

The Sherman and Tomahawk live traps were baited with a mixture of cornmeal, banana, oatmeal, peanut butter, and cod liver oil, or with pieces of banana and bacon. All traps were monitored daily.

The bats in each area of Serra da Jiboia and Pratigi APA were sampled with a variable number of mist nets of 9 or $12 \mathrm{~m}$ in length and three meters high, with a mesh of $20 \mathrm{~mm}$. At least five nets were used, but in some areas ten nets were mounted if the local conditions were favorable. The total sampling effort (Straube \& Bianconi, 2002) in Serra da jiboia was 21,581 $\mathrm{m} 2$ of mist net-hour and in Pratigi APA 20,448 $\mathrm{m}^{2}$ of mist net-hour. 
The nets were mounted next to watercourses, within the forest fragments or at their edges. The nests were opened in the evening, at around 6 p.m., and closed at 10 p.m., if the weather permitted sampling throughout the entire interval. The occurrence of captured individuals was checked every 20 minutes. A few bats were captured with dip nets in natural (caves, for example) or artificial shelters (such as human edifications near the forest).

To capture the small mammals in areas of the Serra da Jiboia and the Pratigi APA, 15 capture sessions were held between August 2013 and March 2017, each lasting for a period of at least seven consecutive days, resulting in an effort of 54,474 capture traps, distributed as follows: Serra da Jiboia: 37,758 traps (Sherman and Tomahawk) and 3,164 buckets; Pratigi APA: 13,552 traps (Sherman and Tomahawk).

For the areas of Ilhéus, Una, Mascote, and Belmonte, there were three capture sessions between June and December 2015-2016, each period consisting of 10 consecutive nights, totaling 13,950 capture traps (5,400 Sherman, 5,400 Tomahawk and 3,150 pitfall traps), throughout the study period.

The captured individuals were measured and weighed for identification (mainly using Reis et al. 2013; 2014), with record of sex and age group, defined according to dental eruptions of upper molars (Graipel et al., 2006; Bergallo, 1995) as young and adults. Normally, a token couple was collected for most of the species and deposited in the Collection of Mammals at the Museum of Zoology of the State University of Feira de Santana (under the code MZFS DM).

\section{Collection of biological material}

The procedures for collecting the specimens were carried out following the ethical principles of the Brazilian College of Animal Experimentation and the Federal Council of Veterinary Medicine (CFMV, 2012), with system authorizations and federal agencies authorization (SISBIO), under numbers 17131-4, 43948-1 and 41440-1, and according to the principles of the Board of Ethics in Animal Use of the State University of Santa Cruz (No 003/2013) and the State University of Feira de Santana (No 39/2017) - CEUA. The samples were collected from the animals deposited in the Alexandre Rodrigues Ferreira Mammalian Collection of the State University of Santa Cruz (CMARFUESC) and in the Collection of Mammals of the Museum of Zoology of the State University of Feira de Santana (MZFS DM). Collections carried out between 2008 and 2013, specifically from Serra da Jibóia and the Pratigi APA, refer to mammalian samples taken by the Museum of Zoology, whose samples were deposited in the Mammal Collection. Access to these samples was authorized by the Museum Mammal Division (Consent of July 8, 2016), endorsed by CEUA Official Letter 39/2017.

Blood samples were obtained through cardiac puncture at the time of measuring the biometrics of the animals. The blood samples were centrifuged, and the sera obtained were aliquoted into $1.5 \mathrm{ml}$ tubes and stored at $-4^{\circ} \mathrm{C}$. Euthanasia was performed through intramuscular administration of ketamine hydrochloride $(30 \mathrm{mg} / \mathrm{kg}$ ) associated with xylazine hydrochloride ( $2 \mathrm{mg} / \mathrm{kg}$ ), according to Cubas et al. (2006). There was also collection of organs and tissues with the objective of compiling scientific collections and serving as material for future research. Later, the animals were identified as to species, sex and age (Bonvicino et al., 2008).

\section{Serological diagnosis}

For the presence of antibodies against T. gondii the Modified Agglutination Test (MAT) was used, using formalin inactivated tachyzoites and 2-mercaptoethanol as described by Dubey \& Desmonts (1987). The cut-off point used was 1:25 and the positive samples were end titrated using 2-fold serial dilutions (Gennari et al., 2015).

\section{Statistical Analysis}

Absolute and relative frequency of serological results were calculated according order of mammals, sex and age group, as by collection areas, with respective $95 \%$ confidence interval for relative frequency. Pearson Chi-square test or Fisher Exact Test were performed to assigned independency of serological results and order of mammals, sex and age group, with calculation of odds ratio and respective $95 \%$ confidence interval when appropriate using Epi Info ${ }^{\text {TM }}$ 7.2.0.1 software (CDC, 2002). All statistical were calculated to a significance level of 5\% 


\section{Results}

Samples were obtained from 368 animals, these being 246 rodents (66.8\%) of 26 distinct species, 104 marsupials (28.2\%) of 12 species and 18 Chiroptera (4.8\%) of 12 species. The total frequency of anti-T. gondii antibodies found was 10.6\% (39/368; CI95\%: $7.86-14.16)$. In rodents this value was 8.5\% (21/246; CI95\%: $5.67-12.71)$, in marsupials 16.3\% (17/104; CI 95\%: 10.48 - 24.19), and in bats 5.5\% (1/18; CI95\%: 1.30 - 26.06), with no differences when the comparison of positive frequencies was performed considering all orders (Table 1). However, when considering the orders Rodentia and Didelmorphia, there was a significant difference with $p=0.0377$ by Fisher's Exact Test and OR=2.0936 (CI95\%: 1.0547-4.1560).

For the variables age group and sex, there was no difference in the frequency of positivity to serology, either in rodents or marsupials $(p>0.05)$. For rodents there was a significant association between the serological result and the place of capture $(p<0.01)$, positives being 6.27 times most likely to happen among animals caught in the Cabruca areas (Table 2). Statistics were not calculated for the Chiroptera due to the low number of positive samples $(n=1)$.

Among the rodents, six species were seropositive: Akodon cursor, Cerradomys subflavus, Hylaeamys laticeps, Oligoryzomys nigripes, Rattus rattus, Thaptomys nigrita, and Trinomys setosus. Six species of marsupials were also seropositive: Didelphis aurita, Gracilinanus microtarsus, G. agilis, Marmosa murina, Marmosops incanus, and Monodelphis americana. Among the Chiroptera, Rhynchonycteris spp. was the only seropositive result. Antibody titers varied between 25 and 50 for rodents (Table 3), between 25 and 400 for marsupials (Table 4) and were 25 for bats (Table 5), with titer 25 being the most frequent in all species (47.37\% of all positive samples).

In relation to origin, $257(70.0 \%)$ small mammals were captured in forest fragments and 110 (29.9\%) in agroforestry cocoa systems (Cabruca). Rodents, marsupials, and bats were caught in 25, 26 and 4 of the 27 studied areas, respectively. Seropositive animals were found in 51.8\% (14/27) of the capture sites (Table 3, 4 and 5). For rodents and marsupials, seropositive animals were found more frequently in collection areas in the South and Far South, with a higher frequency of positives for animals collected in the Cabruca area (municipality of Ilhéus). The only seropositive bat was captured in the Serra da Jibóia area, municipality of Varzedo.

Table 1. Relative frequency (\%), absolute frequency (Pos/Total) and confidence interval 95\% (CI95\%) of wild mammals positive for the presence of anti-Toxoplasma gondii antibodies in fragments of Atlantic Forest of the state of Bahia, according to the order by the exact test of Fisher.

\begin{tabular}{cccc}
\hline Order & \% (Positive/Total) & Cl95\% & P-Value \\
\hline Rodentia & $8.54(21 / 246)$ & $5.67-12.71$ & 0.0886 \\
Didelmorphia & $16.35(17 / 104)$ & $10.48-24.19$ & $1.30-26.03$ \\
Quiroptera & $5.56(1 / 18)$ & 1.30 & \\
\hline
\end{tabular}

Table 2. Relative frequency (\%), absolute frequency (Pos/Total) and confidence interval 95\% (CI95\%) of rodents and marsupials positive for the presence of anti-Toxoplasma gondii antibodies in fragments of Atlantic Forest of Bahia state, second sex, age group and place of capture by the Chi Square Test.

\begin{tabular}{|c|c|c|c|c|c|c|}
\hline \multirow{2}{*}{ Variables } & \multicolumn{3}{|c|}{ Rodents } & \multicolumn{3}{|c|}{ Marsupials } \\
\hline & \% (Pos/Total) & Cl 95\% & P Value & $\%$ (Pos/Total) & Cl $95 \%$ & P Value \\
\hline \multicolumn{7}{|l|}{ Sex } \\
\hline Male & $10.46(16 / 153)$ & $6.56-16.32$ & 0.30 & $17.86(10 / 56)$ & $10.05-29.90$ & 0.53 \\
\hline Female & $5.68(5 / 88)$ & $2.51-12.62$ & & $11.36(5 / 44)$ & $5.05-24.05$ & \\
\hline \multicolumn{7}{|l|}{ Age group } \\
\hline Adult & $8.33(15 / 180)$ & $5.14-13.30$ & 0.67 & $17.19(11 / 64)$ & $9.92-28.26$ & 0.60 \\
\hline Young & $11.76(6 / 51)$ & $5.59-23.44$ & & $11.11(4 / 36)$ & $4.54-25.42$ & \\
\hline \multicolumn{7}{|l|}{ Areas } \\
\hline Cabruca & $17.39(16 / 92)$ & $11.02-26.43$ & $<0.01 *$ & $27.78(5 / 18)$ & $12.57-51.20$ & 0.17 \\
\hline Atlantic Forest & $3.25(5 / 154)$ & $1.43-7.37$ & & $13.95(12 / 86)$ & $8.20-22.85$ & \\
\hline
\end{tabular}

${ }^{*} \mathrm{OR}=6.27(95 \% \mathrm{Cl}: 2.21-17.77)$. The sum is less than the number of samples as some animals have not been registered regarding sex and age. 
Table 3. Absolute frequency ( $N$ ) of animals examined, and absolute frequency $(P)$ and relative frequency (\%) of wild mammals positive for the presence of anti-Toxoplasma gondii antibodies in Modified agglutination test (MAT) in fragments of Atlantic Forest of Bahia State, according to Orders, Species and collection points.

\begin{tabular}{|c|c|c|c|c|c|c|c|c|c|c|c|c|c|c|}
\hline \multirow[b]{2}{*}{ Species } & \multicolumn{2}{|c|}{$\begin{array}{l}\text { Serra da } \\
\text { Jiboia }\end{array}$} & \multicolumn{4}{|c|}{ Pratigi APA } & \multicolumn{2}{|c|}{ South } & \multicolumn{2}{|c|}{ Far South } & \multirow[b]{2}{*}{$\mathbf{N}$} & \multirow[b]{2}{*}{$\mathbf{P}$} & \multirow[b]{2}{*}{$\%$} & \multirow[b]{2}{*}{$\begin{array}{c}\text { MAT } \\
\text { titulation }\end{array}$} \\
\hline & $\begin{array}{l}\frac{0}{0} \\
\frac{0}{0} \\
\frac{d}{\Sigma} \\
\frac{0}{n} \\
\frac{0}{\omega}\end{array}$ & $\begin{array}{l}\frac{0}{0} \\
\frac{N}{N} \\
\frac{N}{5}\end{array}$ & 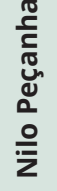 & 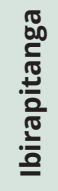 & 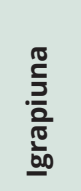 & $\begin{array}{l}\frac{\pi}{2} \\
\frac{0}{3} \\
\underline{\underline{Z}}\end{array}$ & $\stackrel{*}{\stackrel{*}{\varrho}}$ & $\stackrel{\text { }}{5}$ & 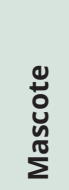 & 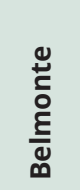 & & & & \\
\hline $\begin{array}{l}\text { Akodon cursor } \\
\text { (Winge. 1887) }\end{array}$ & - & - & - & - & $0 / 20$ & - & $1 / 11$ & & $0 / 1$ & $0 / 1$ & 33 & 1 & 3.03 & $25(1)$ \\
\hline Akodon sp. & - & - & - & - & $0 / 3$ & - & & - & - & - & 3 & 0 & 0.00 & - \\
\hline $\begin{array}{c}\text { Blarinomys } \\
\text { breviceps (Winge. } \\
1887 \text { ) }\end{array}$ & - & - & - & - & - & - & - & $0 / 1$ & - & - & 1 & 0 & 0.00 & - \\
\hline Calomys sp. & - & - & - & - & - & - & - & - & $0 / 2$ & & 2 & 0 & 0.00 & - \\
\hline Cerradomys sp. & - & - & - & - & $0 / 1$ & - & - & - & - & - & 1 & 0 & 0.00 & - \\
\hline $\begin{array}{c}\text { Cerradomys } \\
\text { subflavus (Wagner. } \\
1842 \text { ) }\end{array}$ & - & - & - & - & - & - & $1 / 3$ & - & $0 / 1$ & - & 4 & 1 & 25.00 & $25(1)$ \\
\hline $\begin{array}{l}\text { Cerradomys vivoi } \\
\text { (Percequillo. } \\
\text { Hingst-Zaher. } \\
\text { Bonvicino. 2008) }\end{array}$ & - & - & - & - & $0 / 2$ & - & - & - & - & - & 2 & 0 & 0.00 & - \\
\hline cf. Cerradomys & $0 / 1$ & - & - & - & - & - & - & - & - & - & 1 & 0 & 0.00 & - \\
\hline cf. Hylaeamys & - & - & - & - & $0 / 1$ & - & - & - & - & - & 1 & 0 & 0.00 & - \\
\hline cf. Rhipidomys & $0 / 1$ & - & - & - & - & - & - & - & - & - & 1 & 0 & 0.00 & - \\
\hline $\begin{array}{c}\text { Euryoryzomys } \\
\text { russatus (Wagner. } \\
1848)\end{array}$ & $0 / 1$ & - & - & - & $0 / 1$ & - & - & - & - & - & 2 & 0 & 0.00 & - \\
\hline Euryoryzomys sp. & - & - & - & - & - & - & $0 / 2$ & - & - & $0 / 1$ & 3 & 0 & 0.00 & - \\
\hline Guerlinguetus sp. & - & - & - & - & - & - & - & - & - & $0 / 2$ & 2 & 0 & 0.00 & - \\
\hline $\begin{array}{l}\text { Hylaeamys laticeps } \\
\text { (Lund. 1840) }\end{array}$ & - & - & - & - & $0 / 7$ & - & $12 / 47$ & $0 / 45$ & $0 / 1$ & $0 / 26$ & 126 & 12 & 9.52 & 25 (10). 50 (2) \\
\hline $\begin{array}{l}\text { Necromys lasiurus } \\
\quad \text { (Lund. 1841) }\end{array}$ & - & - & - & - & $0 / 1$ & - & $0 / 1$ & $0 / 1$ & $0 / 1$ & - & 4 & 0 & 0.00 & - \\
\hline $\begin{array}{c}\text { Nectomys } \\
\text { squamipes (Brants. } \\
\text { 1827) }\end{array}$ & - & - & - & - & $0 / 3$ & - & $0 / 4$ & - & - & - & 7 & 0 & 0.00 & - \\
\hline $\begin{array}{c}\text { Oecomys } \\
\text { catherinae } \\
\text { Thomas. } 1909\end{array}$ & - & - & - & - & $0 / 1$ & - & - & $0 / 2$ & - & $0 / 1$ & 4 & 0 & 0.00 & - \\
\hline $\begin{array}{c}\text { Oligoryzomys } \\
\text { nigripes (Olfers. } \\
1818 \text { ) }\end{array}$ & - & - & - & - & - & - & $1 / 6$ & $0 / 3$ & $1 / 1$ & - & 10 & 2 & 20.00 & $25(2)$ \\
\hline $\begin{array}{c}\text { Oxymycterus } \\
\text { dasytrichus } \\
\text { (Schinz. 1821) }\end{array}$ & - & - & - & - & $0 / 4$ & - & - & - & - & - & 4 & 0 & 0.00 & - \\
\hline Oxymycterus sp. & - & - & - & - & $0 / 1$ & - & - & - & - & - & 1 & 0 & 0.00 & - \\
\hline
\end{tabular}

* Collection points in Ilheus Municipality correspond to Cabruca areas. 
Table 3. Continued...

\begin{tabular}{|c|c|c|c|c|c|c|c|c|c|c|c|c|c|c|}
\hline \multirow[b]{2}{*}{ Species } & \multicolumn{2}{|c|}{$\begin{array}{l}\text { Serra da } \\
\text { Jiboia }\end{array}$} & \multicolumn{4}{|c|}{ Pratigi APA } & \multicolumn{2}{|c|}{ South } & \multicolumn{2}{|c|}{ Far South } & \multirow[b]{2}{*}{$\mathbf{N}$} & \multirow[b]{2}{*}{$\mathbf{P}$} & \multirow[b]{2}{*}{$\%$} & \multirow[b]{2}{*}{$\begin{array}{c}\text { MAT } \\
\text { titulation }\end{array}$} \\
\hline & 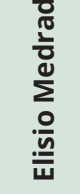 & $\begin{array}{l}\frac{0}{0} \\
\stackrel{d}{N} \\
\frac{N}{7}\end{array}$ & 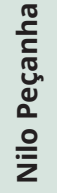 & 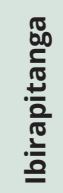 & $\frac{\sqrt{00}}{\frac{0}{0}}$ & $\begin{array}{l}\frac{\pi}{2} \\
\stackrel{0}{2} \\
\underline{\underline{Z}}\end{array}$ & 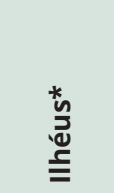 & $\stackrel{\pi}{5}$ & 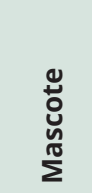 & 롣 & & & & \\
\hline $\begin{array}{c}\text { Rattus rattus } \\
\text { (Linnaeus. 1758) }\end{array}$ & - & - & - & - & - & - & $1 / 1$ & - & - & - & 1 & 1 & 100.00 & - \\
\hline $\begin{array}{c}\text { Rhipidomys } \\
\text { mastacalis (lund. } \\
\text { 1840) }\end{array}$ & - & - & - & - & - & - & $0 / 14$ & $0 / 3$ & $0 / 1$ & $0 / 2$ & 20 & 0 & 0.00 & - \\
\hline $\begin{array}{c}\text { Thaptomys nigrita } \\
\text { (Lichtenstein. } \\
\text { 1829) }\end{array}$ & - & - & - & - & - & - & $0 / 2$ & $1 / 4$ & - & $2 / 3$ & 9 & 3 & 33,33 & $25(3)$ \\
\hline Thrichomys sp & - & - & - & - & - & - & $0 / 1$ & - & - & - & 1 & 0 & 0,00 & - \\
\hline $\begin{array}{c}\text { Trinomys cf. } T . \\
\text { setosus }\end{array}$ & $0 / 1$ & - & - & - & - & - & - & - & - & - & 1 & 0 & 0,00 & - \\
\hline $\begin{array}{l}\text { Trinomys setosus } \\
\text { (Desmarest. 1817) }\end{array}$ & - & $1 / 2$ & - & - & - & - & - & - & - & - & 2 & 1 & 50,00 & \\
\hline TOTAL & $\begin{array}{l}0.00 \\
(0 / 4)\end{array}$ & $\begin{array}{l}50.00 \\
(1 / 2)\end{array}$ & - & - & $\begin{array}{c}0.00 \\
(0 / 45)\end{array}$ & - & $\begin{array}{c}17.39 \\
(16 / 92)\end{array}$ & $\begin{array}{c}1.69 \\
(1 / 59)\end{array}$ & $\begin{array}{l}12.50 \\
(1 / 8)\end{array}$ & $\begin{array}{c}5.56 \\
(2 / 36)\end{array}$ & 246 & 21 & 8,54 & \\
\hline
\end{tabular}

* Collection points in Ilheus Municipality correspond to Cabruca areas.

Table 4. Absolute frequency $(\mathrm{N})$ of animals examined, and absolute frequency $(\mathrm{P})$ and relative frequency (\%) of Didelmorphia positive for the presence of anti-Toxoplasma gondii antibodies in Modified Agglutination Test (MAT) in fragments of Atlantic Forest of Bahia State, according to Species and collection points.

\begin{tabular}{|c|c|c|c|c|c|c|c|c|c|c|c|c|c|c|}
\hline \multirow[b]{2}{*}{ Species } & \multicolumn{2}{|c|}{$\begin{array}{l}\text { Serra da } \\
\text { Jiboia }\end{array}$} & \multicolumn{4}{|c|}{ Pratigi APA } & \multicolumn{2}{|c|}{ South } & \multicolumn{2}{|c|}{ Far South } & \multirow[b]{2}{*}{$\mathbf{N}$} & \multirow[b]{2}{*}{$\mathbf{P}$} & \multirow[b]{2}{*}{$\%$} & \multirow[b]{2}{*}{$\begin{array}{c}\text { MAT } \\
\text { titulation }\end{array}$} \\
\hline & 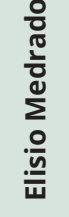 & $\begin{array}{l}\text { 용 } \\
\text { N } \\
\text { N }\end{array}$ & 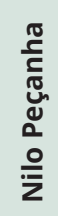 & 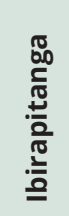 & $\frac{\sqrt{00}}{\frac{00}{20}}$ & 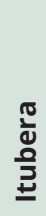 & 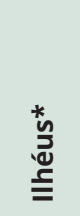 & $\stackrel{\text { క }}{5}$ & 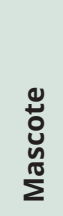 & 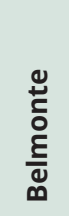 & & & & \\
\hline $\begin{array}{c}\text { Didelphis aurita } \\
\text { (Wied-Neuwied. } \\
\text { 1826) }\end{array}$ & $1 / 1$ & - & - & - & $1 / 3$ & - & $0 / 2$ & $0 / 4$ & $0 / 1$ & - & 11 & 2 & 18.18 & $25(2)$ \\
\hline $\begin{array}{c}\text { Gracilinanus } \\
\text { microtarsus } \\
\text { (Wagner. 1842) }\end{array}$ & - & $0 / 1$ & - & - & - & - & $1 / 1$ & - & - & - & 1 & 1 & 100.00 & $400(1)$ \\
\hline $\begin{array}{c}\text { Gracilinanus } \\
\text { agilis } \\
\text { (Burmeister. } \\
\text { 1854) }\end{array}$ & - & - & - & - & - & - & - & - & $1 / 3$ & $2 / 2$ & 5 & 3 & 60.00 & 25 (2). $400(1)$ \\
\hline $\begin{array}{l}\text { Gracilinanus cf. } \\
\text { G. microtarsus }\end{array}$ & - & - & - & - & - & - & - & - & - & - & 1 & 0 & 0.00 & - \\
\hline $\begin{array}{c}\text { Marmosa } \\
\text { demerarae } \\
\text { (Thomas. 1905) }\end{array}$ & - & - & - & - & - & - & - & $0 / 9$ & - & - & 9 & 0 & 0.00 & - \\
\hline $\begin{array}{l}\text { Marmosa murina } \\
\text { (Linnaeus. 1758) }\end{array}$ & $1 / 1$ & - & - & - & $0 / 4$ & - & $4 / 10$ & $0 / 19$ & $0 / 4$ & $1 / 6$ & 44 & 6 & 13.64 & $\begin{array}{c}25 \text { (3). } 100(2) \\
200(1)\end{array}$ \\
\hline
\end{tabular}

*Collection points in Ilheus Municipality correspond to Cabruca areas. 
Table 4. Continued...

\begin{tabular}{|c|c|c|c|c|c|c|c|c|c|c|c|c|c|c|}
\hline \multirow[b]{2}{*}{ Species } & \multicolumn{2}{|c|}{$\begin{array}{c}\text { Serra da } \\
\text { Jiboia }\end{array}$} & \multicolumn{4}{|c|}{ Pratigi APA } & \multicolumn{2}{|c|}{ South } & \multicolumn{2}{|c|}{ Far South } & \multirow[b]{2}{*}{$\mathbf{N}$} & \multirow[b]{2}{*}{$\mathbf{P}$} & \multirow[b]{2}{*}{$\%$} & \multirow[b]{2}{*}{$\begin{array}{c}\text { MAT } \\
\text { titulation }\end{array}$} \\
\hline & 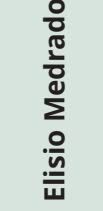 & $\begin{array}{l}\frac{0}{0} \\
\frac{d}{N} \\
\frac{\pi}{7}\end{array}$ & 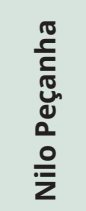 & $\begin{array}{l}\sqrt[5]{00} \\
\frac{0}{0} \\
\stackrel{0}{0} \\
\frac{0}{0} \\
. \frac{0}{0} \\
0\end{array}$ & $\frac{\sqrt{00}}{\frac{00}{50}}$ & $\begin{array}{l}\text { 밈 } \\
\stackrel{0}{3} \\
\underline{\underline{D}}\end{array}$ & 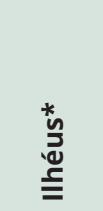 & $\stackrel{\pi}{5}$ & 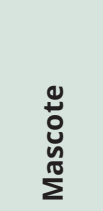 & $\stackrel{\stackrel{\Xi}{\check{0}}}{\stackrel{\Xi}{\varrho}}$ & & & & \\
\hline $\begin{array}{c}\text { Marmosops } \\
\text { incanus (Lund. } \\
\text { 1840) }\end{array}$ & - & - & - & - & $1 / 8$ & $0 / 1$ & $0 / 2$ & - & $1 / 3$ & - & 14 & 2 & 14.29 & $25(2)$ \\
\hline Marmosops sp & - & - & - & - & - & - & - & - & $0 / 1$ & $0 / 1$ & 2 & 0 & 0.00 & \\
\hline $\begin{array}{l}\text { Metachirus } \\
\text { nudicaudatus } \\
\text { (Geoffroy. } \\
\text { 1803) }\end{array}$ & - & - & $0 / 1$ & - & $0 / 2$ & - & - & - & - & - & 3 & 0 & 0.00 & - \\
\hline $\begin{array}{c}\text { Micoureus } \\
\text { paraguayanus } \\
\text { (Tate. 1931) }\end{array}$ & $0 / 1$ & - & - & $0 / 1$ & $0 / 1$ & - & - & - & - & - & 3 & 0 & 0.00 & - \\
\hline $\begin{array}{l}\text { Monodelphis } \\
\text { americana } \\
\text { (Müller. 1776) }\end{array}$ & - & - & - & & $1 / 3$ & - & $0 / 3$ & $1 / 3$ & - & $0 / 1$ & 10 & 3 & 30.00 & $25(3)$ \\
\hline Monodelphis sp. & - & - & - & - & - & - & - & - & - & - & 1 & 0 & 0.00 & - \\
\hline TOTAL & $\begin{array}{c}66.67 \\
(2 / 3)\end{array}$ & $\begin{array}{l}0.00 \\
(0 / 1)\end{array}$ & $\begin{array}{l}0.00 \\
(0 / 1)\end{array}$ & $\begin{array}{l}0.00 \\
(0 / 1)\end{array}$ & $\begin{array}{l}14.29 \\
(3 / 21)\end{array}$ & $\begin{array}{l}0.00 \\
(0 / 1)\end{array}$ & $\begin{array}{l}27.78 \\
(5 / 18)\end{array}$ & $\begin{array}{c}2.86 \\
(1 / 35)\end{array}$ & $\begin{array}{l}16.67 \\
(2 / 12)\end{array}$ & $\begin{array}{l}36,36 \\
(4 / 11)\end{array}$ & 104 & 17 & 16.35 & \\
\hline
\end{tabular}

*Collection points in Ilheus Municipality correspond to Cabruca areas.

Table 5. Absolute frequency $(\mathrm{N})$ of animals examined, and absolute frequency $(\mathrm{P})$ and relative frequency (\%) of Quiroptera positive for the presence of anti-Toxoplasma gondii antibodies in Modified Agglutination Test (MAT) in fragments of Atlantic Forest of Bahia State, according to Species and collection points.

\begin{tabular}{|c|c|c|c|c|c|c|c|c|c|c|c|c|c|c|}
\hline \multirow[b]{2}{*}{ Species } & \multicolumn{2}{|c|}{$\begin{array}{l}\text { Serra da } \\
\text { Jiboia }\end{array}$} & \multicolumn{4}{|c|}{ Pratigi APA } & \multicolumn{2}{|c|}{ South } & \multicolumn{2}{|c|}{ Far South } & \multirow[b]{2}{*}{$\mathbf{N}$} & \multirow[b]{2}{*}{$\mathbf{P}$} & \multirow[b]{2}{*}{$\%$} & \multirow[b]{2}{*}{$\begin{array}{l}\text { MAT } \\
\text { titulation }\end{array}$} \\
\hline & 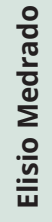 & $\begin{array}{l}\frac{0}{0} \\
\text { N } \\
\frac{1}{\pi}\end{array}$ & 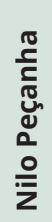 & 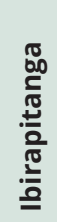 & $\begin{array}{l}\frac{0}{5} \\
\frac{5}{2} \\
\frac{0}{0} \\
\frac{0}{00}\end{array}$ & $\begin{array}{l}\frac{\pi}{ \pm !} \\
\frac{0}{3} \\
\underline{ \pm}\end{array}$ & 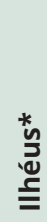 & $\stackrel{0}{5}$ & 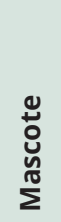 & 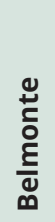 & & & & \\
\hline $\begin{array}{l}\text { Anoura geoffroyi } \\
\text { (Geoffroy. 1838) }\end{array}$ & - & $0 / 1$ & - & - & - & - & - & - & - & - & 1 & 0 & 0.00 & - \\
\hline $\begin{array}{l}\text { Artibeus lituratus } \\
\text { (Olfers. 1818) }\end{array}$ & - & $0 / 3$ & - & - & - & - & - & - & - & - & 3 & 0 & 0.00 & - \\
\hline $\begin{array}{l}\text { Carollia perspicillata } \\
\text { (Linnaeus. 1758) }\end{array}$ & - & $0 / 2$ & - & - & - & - & - & - & - & - & 2 & 0 & 0.00 & - \\
\hline cf. Myotis & - & $0 / 1$ & - & - & - & - & - & - & - & - & 1 & 0 & 0.00 & - \\
\hline $\begin{array}{l}\text { Dermanura cinerea } \\
\text { (gervais. 1856) }\end{array}$ & - & $0 / 1$ & - & - & - & - & - & - & - & - & 1 & 0 & 0.00 & - \\
\hline $\begin{array}{c}\text { Desmodus rotundus } \\
\text { (Geoffroy. 1810) }\end{array}$ & - & $0 / 2$ & - & - & - & - & - & - & - & - & 2 & 0 & 0.00 & - \\
\hline $\begin{array}{c}\text { Glossophaga } \\
\text { soricina (Pallas. } \\
1766)\end{array}$ & - & - & - & - & $0 / 1$ & - & - & - & - & - & 1 & 0 & 0.00 & - \\
\hline
\end{tabular}

*Collection points in Ilheus Municipality correspond to Cabruca areas. 
Table 5. Continued...

\begin{tabular}{|c|c|c|c|c|c|c|c|c|c|c|c|c|c|c|}
\hline \multirow[b]{2}{*}{ Species } & \multicolumn{2}{|c|}{$\begin{array}{l}\text { Serra da } \\
\text { Jiboia }\end{array}$} & \multicolumn{4}{|c|}{ Pratigi APA } & \multicolumn{2}{|c|}{ South } & \multicolumn{2}{|c|}{ Far South } & \multirow[b]{2}{*}{$\mathbf{N}$} & \multirow[b]{2}{*}{$\mathbf{P}$} & \multirow[b]{2}{*}{$\%$} & \multirow[b]{2}{*}{$\begin{array}{l}\text { MAT } \\
\text { titulation }\end{array}$} \\
\hline & 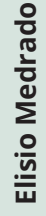 & $\begin{array}{l}\frac{0}{0} \\
\frac{d}{N} \\
\frac{1}{>}\end{array}$ & 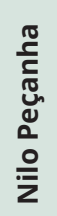 & 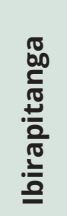 & $\frac{\sqrt{00}}{\frac{00}{0}}$ & $\begin{array}{l}\frac{\pi}{2} \\
\frac{0}{2} \\
\underline{\underline{2}}\end{array}$ & 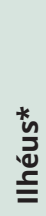 & $\stackrel{\pi}{5}$ & 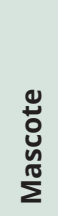 & 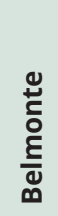 & & & & \\
\hline $\begin{array}{c}\text { Phylloderma } \\
\text { stenops (Peters. } \\
\text { 1875) }\end{array}$ & - & - & - & - & $0 / 1$ & - & - & - & - & - & 1 & 0 & 0.00 & - \\
\hline $\begin{array}{c}\text { Platyrrhinus } \\
\text { lineatus (Geoffroy. } \\
\text { 1810) }\end{array}$ & - & $0 / 2$ & - & - & - & - & - & - & - & - & 2 & 0 & 0.00 & - \\
\hline Rhynchonycteris sp. & - & $1 / 2$ & - & - & - & - & - & - & - & - & 2 & 1 & 50.00 & $25(1)$ \\
\hline $\begin{array}{l}\text { Sturnira lilium } \\
\text { (Geoffroy. 1810) }\end{array}$ & - & $0 / 1$ & - & - & - & - & - & - & - & - & 1 & 0 & 0.00 & - \\
\hline $\begin{array}{l}\text { Sturnira tildae (de } \\
\text { la Torre. 1959) }\end{array}$ & - & $0 / 1$ & - & - & - & - & - & - & - & - & 1 & 0 & 0.00 & - \\
\hline TOTAL & - & $\begin{array}{c}6.25 \\
(1 / 16)\end{array}$ & - & - & $\begin{array}{l}0.00 \\
(0 / 2)\end{array}$ & - & - & - & - & - & 18 & 1 & 5.56 & \\
\hline
\end{tabular}

*Collection points in Ilheus Municipality correspond to Cabruca areas.

\section{Discussion}

In this study, the results show a greater occurrence of anti-T. gondii antibodies in marsupials (16.5\%; Cl95\%: 10.59 - 24.88) than in rodents (8.5\%; CI95\%: 5.67 - 12.71) and bats (5.5\%; CI95\%: 1.30 - 26.03), with a significant difference between marsupials and rodents $(p=0.0377)$. Gennari et al. (2015), using the same diagnostic technique and cut-off point adopted in this study, in areas of Atlantic Forest in municipalities in the state of São Paulo-SP, did not find differences in antibody rates between the studied orders.

The higher frequency of seropositive marsupials in this study can be explained by the different habits of the animals, the habitat, the sample size, the serological test that was used, the cutoff point or the presence of the definitive hosts (Tenter et al., 2000; Siqueira et al., 2013). According to Ferraroni \& Marzochi (1980), a greater prevalence of infection in marsupials may be related to dietary habits that facilitate infection through food ingestion, since the captured animals have a predominantly omnivorous diet (Paglia et al., 2012).

This is the first report of anti-T. gondii antibodies in T. nigrita, H. laticeps, and C. subflavus rodents, in M. americana, G. microtarsus, G. agilis, and M. incanus marsupials, and in bats of the genus Rhynchonycteris. Perhaps these species had never been tested for anti-T. gondii antibodies, except for the rodent C. subflavus, which had already been investigated using MAT, albeit with a negative result (Siqueira et al., 2013).

All positive rodents are terrestrial species, which inhabit forest and vegetable formations (Bonvicino et al., 2008). Terrestrial mammals are significantly more exposed to $T$. gondii than scansorial or arboreal species, so this habitat may contribute to the increased likelihood of infection with oocysts of $T$. gondii present in the soil or in contaminated water. As such, in the wild, the vertical use of space probably reduces the risk of infection by T. gondii (de Thoisy et al., 2003).

The serological results of the Thrichomys sp. species were negative, however, studies carried out in the Atlantic Forest of northeast and southeastern Brazil have reported the presence of anti-T. gondii antibodies in species of this genus, with titration varying between 25 and 6400 (Gennari et al., 2015; Siqueira et al., 2013). In the present study, the negative result in these species may be related to the low number of animals analyzed when compared to the mentioned studies, in which the number of individuals tested was greater than 100.

The rodent Oligoryzomys nigripes was first described as seropositive for $T$. gondii in a study conducted by Gennari et al. (2015) in southeastern Brazil. In this study the seroprevalence in this species reached 20\% (2/10). These animals are generalist species, being found within forests and open vegetation formations with great adaptability 
to anthropic environments, including agricultural areas (Bonvicino et al., 2008). This characteristic explains the fact that $60 \%(6 / 10)$ of the individuals captured from 0 . nigripes occurred in areas of Cabruca. This adaptation to areas close to human habitation and domestic animals (Püttker et al., 2008) makes this species important in the epidemiology of $T$. gondii, as it can serve as a source of infection for domestic and feral cats.

The seroprevalence of T. gondii in marsupials of the genus Marmosa ("cuícas") varied between 0\% (0/9) in Marmosa demerarae and 13.95\% (6/43) in Marmosa murina. In Brazil, the presence of anti-T. gondii antibodies in these species has already been described in research using MAT by Siqueira et al. (2013) that found a lower occurrence of T. gondii antibodies in Marmosa murina and Marmosa demerarae, with 2.3\% (1/43) positive animals in both species.

This study describes the first report of anti-T. gondii antibodies in Monodelphis americana with 33.33\% (3/9) positive animals. However, previous studies detected anti-T. gondii antibodies in specimens of the same genus, Monodelphis domestica, which presented a frequency of infection between $10(1 / 10)$ and 25\% (1/4), in studies carried out in the states of Pernambuco and Rio Grande do Norte, respectively (Fournier et al., 2014; Siqueira et al., 2013).

Opossum (Didelphis aurita) presented $18.1 \%(2 / 11)$ positive serology for $T$. gondii. This genus is commonly reported as reagent in research involving small wild mammals (Salata et al., 1985; Yai et al. 2003; Fornazari et al., 2011; Vitaliano et al., 2014; Gennari et al., 2015). Marsupials of the genus Didelphis have a mostly synanthropic habitat with great adaptive efficiency to various types of habitats, including large urban centers (Bonvicino et al., 2008; Abreu, 2013). The proximity to human populations promotes hunting of these animals for meat consumption and even for medicinal use (Azevedo \& Barros, 2014), making these animals potential transmitters of toxoplasmosis and other zoonoses such as leishmaniasis and rabies (Santiago et al., 2007).

The genus of serology positive Chiroptera is represented by a species with insectivorous food habit, which, according to Graczyk et al. (2005) may pose an infection risk to these animals, as insects can house viable oocysts in their gastrointestinal tract.

A possible explanation for the low antibody titration may be the fact that the animals were captured at the initial stage of infection, before the production of IgG, detectable by the technique used. The MAT detects only IgG antibodies; therefore, it may give false negative results during early stages of acute infection (Dubey, 2010). This possibility should be taken into consideration when using serological techniques, because magnitude of the response varies according to the strain and stage of T. gondii inoculated (Dubey \& Frenkel, 1998; Caldart et al., 2017). However, MAT has shown superior sensitivity when compared to other serological tests such as the latex agglutination test (LAT), the indirect hemagglutination test (IHAT), the enzyme linked immunosorbent assay (ELISA), and the indirect fluorescent antibody test (IFAT) (Dubey et al., 1995; Mainar-Jaime \& Barberán, 2007; Shaapan et al., 2008). Besides the high sensitivity, the possibility of presenting nonspecific reactions is low. In a study by Dubey (1997), serum samples from $T$. gondii-negative pigs were infected with protozoa, helminths, and viruses and evaluated for the cross reaction of anti- $T$ gondii antibodies using MAT, and no evidence of cross reacting with other organisms was found. The areas of Cabruca presented greater prevalence of positive Rodents than the forest fragments $(p=1)$, being places with greater anthropic pressure, more intense environmental degradation and lower biodiversity enabling a close relationship of the wild animals with humans, and domestic animals (Paglia et al., 2012). This interaction can cause increased competition for natural and anthropogenic environments, in addition to resulting in the transmission of diseases from one group to the other (Ogrzewalska et al., 2011).

In the studied region there are species of wild cats, Leopardus pardalis, Leopardus wiedii, Panthera onca and Puma concolor (Moura, 2003), that might play role in the zoonotic cycle of $T$. gondii being the define host. However, no studies on the occurrence of antibodies to $T$. gondii in wild felids in Bahia is available.

Studies of toxoplasmosis in the wild environment enables the production of knowledge related to the epidemiology of toxoplasmosis in different environments. However, difficulties in obtaining samples and insufficient knowledge of the biology, ecology, and ethology of the potential reservoirs limit such investigations (de Thoisy et al., 2003).

Although research indicates that wild animals are often positive in serological tests for $T$. gondii, the role of these species in the epidemiological cycle of the disease is not well known (Dubey, 2010). As such, the study of parasites that can be transmitted by wild mammals is necessary to identify the possible risks to the human population and, consequently, to enable the adoption of control and prevention measures, in regard to the importance of $T$. gondii for One Health approaches.

\section{Conclusion}

Results indicate the presence of antibodies to $T$. gondii in the wild animals, with the mammals playing a role in the epidemiological cycle of toxoplasmosis, and possibly being responsible for transmission of the parasite to 
its definitive host and, thus, enabling parasite maintenance in the environment, especially on regions under high anthropogenic effect.

\section{Acknowledgements}

This work was supported in part by the Coordenação de Aperfeiçoamento de Pessoal de Nível Superior - Brasil (CAPES) - Finance Code 001, Fundação de Amparo a Pesquisa do Estado da Bahia (FAPESB) (grant PNE0001/2014), and Conselho Nacional de Desenvolvimento Científico e Tecnológico (CNPq) (grant 306308/2015-0).

\section{References}

Abreu JAP, Krawczak FS, Nunes FP, Labruna MB, Pena HFJ. Anti-Toxoplasma gondii and anti-Neospora caninum antibodies in capybaras (Hydrochoerus hydrochaeris) from Itu Municipality, São Paulo. Rev Bras Parasito/ Vet 2016; 25(1): 116-118. http://dx.doi. org/10.1590/S1984-29612016002. PMid:26982562.

Abreu MC. Evidência de sinurbização do sariguê (Didelphis) no ecossistema urbano de Feira de Santana (BA): Ocorrência e interação com os seres humanos [dissertation]. Feira de Santana: Universidade Estadual de Feira de Santana; 2013.

Azevedo PA, Barros FB. Comida, remédio, renda: conhecimentos e usos da mucura (Didelphis marsupialis) por comunidades ribeirinhas da várzea amazônica. Amazônica -. Rev Antropol 2014; 5(3): 862. http://dx.doi.org/10.18542/amazonica.v5i3.1608.

Bahia. Superintendência de Estudos Econômicos e Sociais do Estado da Bahia. Sistemas de Informações Municipais [online]. Salvador: SEl; 2015 [cited 2015 Sep 23]. Available from: http://sim.sei.ba.gov.br/sim/informaçoes_municipais.wsp

Bergallo HG. Comparative life-history characteristics of two species of rats, Proechimys iheringi and and Oryzomys intermedius, in an Atlantic Forest of Brazil. Mammalia 1995; 59(1): 51-64. http://dx.doi.org/10.1515/mamm.1995.59.1.51.

Bonvicino CR, Oliveira JA, D'Andrea PS. Guia dos roedores do Brasil, com chaves para gêneros baseadas em caracteres externos. Rio de Janeiro: Centro Pan-Americano de Febre Aftosa-OPAS/OMS; 2008.

Cabral AD, D’Auria SR, Camargo MC, Rosa AR, Sodré MM, Galvão-Dias MA, et al. Seroepidemiology of Toxoplasma gondii infection in bats from São Paulo city, Brazil. Vet Parasitol 2014; 206(3-4): 293-296. http://dx.doi.org/10.1016/j.vetpar.2014.10.014. PMid:25468027.

Cabral AD, Gama AR, Sodré MM, Savani ES, Galvão-Dias MA, Jordão LR, et al. First isolation and genotyping of Toxoplasma gondii from bats (Mammalia: chiroptera). Vet Parasitol 2013; 193(1-3): 100-104. http://dx.doi.org/10.1016/j.vetpar.2012.11.015. PMid:23200751.

Caldart ET, Freire RL, Ferreira FP, Ruffolo BB, Sbeghen MR, Mareze M, et al. Leishmania in synanthropic rodents (Rattus rattus): new evidence for the urbanization of Leishmania (Leishmania) amazonensis. Rev Bras Parasitol Vet 2017; 26(1): 17-27. http://dx.doi. org/10.1590/s1984-29612017001. PMid:28177041.

Centers for Disease Control and Prevention - CDC. Epi Info [online]. Atlanta: CDC; 2002 [cited 2015 Sep 23]. Available from: https://wwwn.cdc.gov/epiinfo/html/prevVersion.htm

Cola GA, Garcia JL, Costa L, Ruffolo B, Navarro IT, Freire RL. Comparação da reação de imunofluorescência indireta e do teste de aglutinação modificado na detecção de anticorpos anti-Toxoplasma gondii em ratos. Semina: Ciênc Agrár 2010; 31 (3): 717-722. http://dx.doi.org/10.5433/1679-0359.2010v31n3p717.

Conselho Federal de Medicina Veterinária - CFMV. Guia brasileiro de boas práticas para eutanásia em animais - conceitos e procedimentos recomendados. Brasília: Conselho Federal de Medicina Veterinária; 2012.

Costa DG, Marvulo MF, Silva JS, Santana SC, Magalhães FJ, Lima CDF Fo, et al. Seroprevalence of Toxoplasma gondii in domestic and wild animals from the Fernando de Noronha, Brazil. J Parasito/ 2012; 98(3): 679-680. http://dx.doi.org/10.1645/GE-2910.1. PMid:22150091.

Cubas ZS, Silva JC, Dias J. Tratado de animais selvagens: medicina veterinária. São Paulo: Roca; 2006.

de Thoisy B, Demar M, Aznar C, Carme B. Ecologic correlates of Toxoplasma gondii exposure in free-ranging neotropical mammals. J Wildl Dis 2003; 39(2): 456-459. http://dx.doi.org/10.7589/0090-3558-39.2.456. PMid:12910778.

Dubey JP, Desmonts G. Serological responses of equids fed Toxoplasma gondii oocysts. Equine Vet J 1987; 19(4): 337-339. http:// dx.doi.org/10.1111/j.2042-3306.1987.tb01426.x. PMid:3622463.

Dubey JP, Frenkel JK. Toxoplasmosis of rats: a review, with considerations of their value as an animal model and their possible role in epidemiology. Vet Parasitol 1998; 77(1): 1-32. http://dx.doi.org/10.1016/S0304-4017(97)00227-6. PMid:9652380. 
Dubey JP, Thulliez P, Weigel RM, Andrews CD, Lind P, Powell EC. Sensitivity and specificity of various serologic tests for detection of Toxoplasma gondii infection in naturally infected sows. Am J Vet Res 1995; 56(8): 1030-1036. PMid:8533974.

Dubey JP. Toxoplasmosis of animals and humans. Boca Raton: CRC Press; 2010.

Dubey JP. Validation of the specificity of the modified agglutination test for toxoplasmosis in pigs. Vet Parasitol 1997; 71(4): 307310. http://dx.doi.org/10.1016/S0304-4017(97)00016-2. PMid:9299699.

Faria D, Paciencia MLB, Dixo M, Laps RR, Baumgarten J. Ferns, frogs, lizards, birds and bats in forest fragments and shade cacao plantations in two contrasting landscapes in the Atlantic forest, Brazil. Biodivers Conserv 2006; 16(8): 2335-2357. http://dx.doi. org/10.1007/s10531-007-9189-z.

Ferraroni J], Marzochi MCA. Prevalência da infecção pelo Toxoplasma gondii em animais domésticos, silvestres e grupamentos humanos da Amazônia. Mem Inst Oswaldo Cruz 1980; 75(1-2): 99-109. http://dx.doi.org/10.1590/S0074-02761980000100010. PMid:7231162.

Fornazari F, Teixeira CR, Silva RC, Leiva M, Almeida SC, Langoni H. Prevalence of antibodies against Toxoplasma gondii among Brazilian white-eared opossums (Didelphis albiventris). Vet Parasitol 2011; 179(1-3): 238-241. http://dx.doi.org/10.1016/j. vetpar.2011.02.005. PMid:21435790.

Fournier GFSR, Lopes MG, Marcili A, Ramirez DG, Acosta ICL, Ferreira JIGS, et al. Toxoplasma gondii in domestic and wild animals from forest fragments of the municipality of Natal, northeastern Brazil. Rev Bras Parasitol Vet 2014; 23(4): 501-508. http://dx.doi. org/10.1590/s1984-29612014092. PMid:25517529.

Gennari SM, Ogrzewalska MH, Soares HS, Saraiva DG, Pinter A, Nieri-Bastos FA, et al. Toxoplasma gondii antibodies in wild rodents and marsupials from the Atlantic Forest, state of São Paulo, Brazil. Rev Bras Parasitol Vet 2015; 24(3): 379-382. http:// dx.doi.org/10.1590/S1984-29612015045. PMid:26444068.

Graczyk TK, Knight R, Tamang L. Mechanical transmission of human protozoan parasites by insects. Clin Microbiol Rev 2005; 18(1): 128-132. http://dx.doi.org/10.1128/CMR.18.1.128-132.2005. PMid:15653822.

Graipel ME, Cherem JJ, Monteiro-Filho ELA, Glock L. Dinâmica populacional de marsupiais e roedores no Parque Municipal da Lagoa do Peri, Ilha de Santa Catarina, Sul do Brasil. Mastozool Neotrop 2006; 13(1): 31-49.

Horta MC, Ragozo AMA, Casagrande RA, Matushima ER, Souza GO, Morais ZM, et al. Occurrence of anti-Toxoplasma gondii, Neospora caninum and Leptospira spp. antibodies in opossums (Didelphis spp.) in São Paulo State, Brazil. Braz J Vet Res Anim Sci 2016; 53(3): 1-9. https://doi.org/10.11606/issn.1678-4456.bjvras.2016.110381.

Jesus RF, Rodrigues GM, Silva EM, Carneiro AJ, Franke CR, Cunha RM, et al. Toxoplasmatinae parasites in bats from Bahia State, Brazil. J Wildl Dis 2017; 53(1): 144-147. http://dx.doi.org/10.7589/2016-03-065. PMid:27705102.

Mainar-Jaime RC, Barberán M. Evaluation of the diagnostic accuracy of the modified agglutination test (MAT) and an indirect ELISA for the detection of serum antibodies against Toxoplasma gondii in sheep through Bayesian approaches. Vet Parasito/ 2007; 148(2): 122-129. http://dx.doi.org/10.1016/j.vetpar.2007.05.018. PMid:17624672.

Mori SA, Boom BM, Carvalho AM, Santos TS. Souther Bahian Moist forests. Bot Rev 1983; 49(2): 155-232. http://dx.doi.org/10.1007/ BF02861011.

Moura RT. Distribuição e ocorrência de mamíferos na Mata Atlântica do Sul da Bahia. In: Prado PI, Landau EC, Moura RT, Pinto LPS, Fonseca GAB, Alger K. Corredor de biodiversidade da Mata Atlântica do Sul da Bahia. Ilhéus: Instituto de Estudos Sócio Ambientais do Sul da Bahia e Conservation International do Brasil; 2003. p. 1-22.

Myers N, Mittermeier RA, Mittermeier CG, Fonseca GA, Kent J. Biodiversity hotspots for conservation priorities. Nature 2000; 403(6772): 853-858. http://dx.doi.org/10.1038/35002501. PMid:10706275.

Ogrzewalska M, Uezu A, Labruna MB. Ticks (Acari: Ixodidae) infesting wild birds in the Atlantic Forest in northeastern Brazil, with notes on rickettsial infection in ticks. Parasitol Res 2011; 108(3): 665-670. http://dx.doi.org/10.1007/s00436-010-2111-8. PMid:20953629.

Paglia AP, Fonseca GAB, Rylands AB, Herrmann G, Aguiar LMS, Chiarello AG, et al. Annotated checklist of brazilian mammals. Occasional papers in conservation biology. 2nd ed. Arlington: Conservation International; 2012.

Piasentin FB, Saito $\mathrm{CH}$. Os diferentes métodos de cultivo de cacau no sudeste da Bahia, Brasil: aspectos históricos e percepções. Bol Mus Para Emílio Goeldi Ciênc Hum 2014; 9(1): 61-78. http://dx.doi.org/10.1590/S1981-81222014000100005.

Pinto LP, Bedê L, Paese A, Fonseca M, Paglia A, Lamas I. Mata Atlântica Brasileira: os desafios para conservação da biodiversidade de um hotspot mundial. In: Rocha CFG, Bergallo HG, Van Sluys M, Alves MAS, editors. Biologia da conservação: essências. São Carlos: Rima; 2006. p. 69-96.

Püttker T, Pardini R, Meyer-Lucht Y, Sommer S. Responses of five small mammal species to micro-scale variations in vegetation structure in secondary Atlantic Forest remnants, Brazil. BMC Ecol 2008; 8(1): 9. http://dx.doi.org/10.1186/1472-6785-8-9. PMid:18457587. 
Reis NR, Fregonezi MN, Peracchi AL, Shibatta OA. Morcegos do Brasil: guia de campo. Rio de Janeiro: Technical Books Editora; 2013.

Reis NR, Peracchi AL, Rossaneis BK, Fregonezi MN. Técnicas de estudo aplicadas aos mamíferos silvestres brasileiros. 2nd ed. Rio de Janeiro: Technical Books Editora; 2014.

Rendón-Franco E, Xicoténcatl-García L, Rico-Torres CP, Muñoz-García Cl, Caso-Aguilar A, Suzán G, et al. Toxoplasmosis seroprevalence in wild small rodents, potentially preys of ocelots in north-eastern México. Parasite 2014; 21: 57. http://dx.doi. org/10.1051/parasite/2014058. PMid:25375977.

Salata E, Yoshida LA, Pereira EA, Corrêa FMA. Toxoplasmose em animais silvestres e domésticos da região de Botucatu, Estado de São Paulo, Brasil. Rev Inst Med Trop São Paulo 1985; 27(1): 20-22. http://dx.doi.org/10.1590/S0036-46651985000100004. PMid:3898317.

Sangioni LA, Horta MC, Vianna MCB, Gennari SM, Soares RM, Galvão MAM, et al. Rickettsial infection in animals and Brazilian spotted fever endemicity. Emerg Infect Dis 2005; 11(2): 265-270. http://dx.doi.org/10.3201/eid1102.040656. PMid:15752445.

Santiago MEB, Vasconcelos RO, Fattori KR, Munari DP, Michelin AF, Lima VMF. An investigation of Leishmania spp. in Didelphis spp. from urban and peri-urban areas in Bauru (São Paulo, Brazil). Vet Parasito/ 2007; 150(4): 283-290. http://dx.doi.org/10.1016/j. vetpar.2007.09.026. PMid:17996372.

Shaapan RM, El-Nawawi FA, Tawfik MAA. Sensitivity and specificity of various serological tests for the detection of Toxoplasma gondii infection in naturally infected sheep. Vet Parasito/ 2008; 153(3-4): 359-362. http://dx.doi.org/10.1016/j.vetpar.2008.02.016. PMid:18406534.

Siqueira DB, Aléssio FM, Mauffrey JF, Marvulo MFV, Ribeiro VO, Oliveira RL, et al. Seroprevalence of Toxoplasma gondii in Wild Marsupials and Rodents from the Atlantic Forest of Pernambuco State, Northeastern Region, Brazil. J Parasito/ 2013; 99(6): 11401143. http://dx.doi.org/10.1645/GE-2855.1. PMid:23829204.

Straube FC, Bianconi GV. Sobre a grandeza e a unidade utilizada para estimar esforço de captura com utilização de redes-deneblina. Chiroptera Neotrop 2002; 8: 1-2.

Tenter AM, Heckeroth AR, Weiss LM. Toxoplasma gondii: from animals to humans. Int J Parasito/ 2000; 30(12-13): 1217-1258. http://dx.doi.org/10.1016/S0020-7519(00)00124-7. PMid:11113252.

Thomas W. Natural vegetation types in southern Bahia. In: Prado PI, Landau EC, Moura RT, Pinto LPS, Fonseca GAB, Alger K. Corredor de biodiversidade da Mata Atlântica do Sul da Bahia. Ilhéus: Instituto de Estudos Sócio Ambientais do Sul da Bahia e Conservation International do Brasil; 2003. p. 1-4.

Thompson RCA, Lymbery AJ, Smith A. Parasites, emerging disease and wildlife conservation. Int J Parasitol 2010; 40(10): 11631170. http://dx.doi.org/10.1016/j.ijpara.2010.04.009. PMid:20452354.

Thompson RCA. Parasite zoonoses and wildlife: one health, spillover and human activity. Int J Parasitol 2013; 43(12-13): 10791088. http://dx.doi.org/10.1016/j.ijpara.2013.06.007. PMid:23892130.

Vitaliano SN, Soares HS, Pena HFJ, Dubey JP, Gennari SM. Serologic evidence of Toxoplasma gondii infection in wild birds and mammals from southeast Brazil. J Zoo Wildl Med 2014; 45(1): 197-199. http://dx.doi.org/10.1638/2013-0179R.1. PMid:24712186.

Yai LEO, Cañón-Franco WA, Geraldi VC, Summa MEL, Camargo GO, Dubey JP, et al. Seroprevalence of Neospora caninum and Toxoplasma gondii antibodies in the South American opossum (Didelphis marsupialis) from the city of São Paulo, Brazil.J Parasitol 2003; 89(4): 870-871. http://dx.doi.org/10.1645/GE-83R. PMid:14533710. 